\title{
ARTIGOS
}

\section{LA IDENTIDAD NEGADA: HISTORIA Y SUBALTERNIZACIÓN CULTURAL DESDE TESTIMONIOS ESCOLARES MAPUCHE}

OMAR TURRA • DESIDERIO CATRIQUIR • MARIO VALDÉS

\section{RESUMEN}

Este artículo presenta resultados de una investigación que tuvo como propósito indagar en experiencias escolares de personas mapuche, específicamente en sus significaciones respecto de la historia enseñada en su formación escolar. La investigación socioeducativa se desarrolló en perspectiva histórica, utilizando la revisión documental como técnica de producción de información y el análisis de contenido como procedimiento para reducir y categorizar la información relevante. Los resultados muestran experiencias de agravio y discriminación en la historia enseñada, así como expresiones de resistencia a sus construcciones discursivas, todas manifestaciones que permiten comprender el rol estratégico de la historia como disciplina escolar, en el proceso de escolarización, para la construcción de hegemonía/subalternización en el contexto interétnico mapuche/no mapuche.

ENSEÑANZA DE LA HISTORIA • IDENTIDAD • ALUMNOS • CULTURA MAPUCHE

\section{THE DENIED IDENTITY: HISTORY AND CULTURAL SUBALTERNIZATION FROM MAPUCHE STUDENTS' TESTIMONIES}

\section{ABSTRACT}

This article presents the results of a research that explored the school experiences of Mapuche people, specifically as regards their significance for taught history in their schooling process. The socio-educational research was developed within a historical framework, employing archival review as an information-producing technique, and content analysis as a procedure to reduce and categorize the relevant information. Results show grievance and discrimination episodes in taught history, as well as expressions of resistance to the discursive constructions of the latter. These are all manifestations that cast light on the strategic role of history as a school subject, in the schooling process, to build hegemony/subalternization in the Mapuche/non-Mapuche interethnic context. 
RÉSUMÉ

Cet article présente résultats d'une recherche dont le but a été d'analyser des expériences scolaires de personnes mapuche, plus particulièrement en ce qui concerne leur rapport à l'enseignement de l'histoire tout au long de leur formation scolaire. Cette étude socio-éducative s'est développée dans une perspective historique, en utilisant la révision des documents comme technique de production de l'information et l'analyse de contenu comme procédé afin de cibler et catégoriser les informations pertinentes. Les résultats montrent des expériences de grief et de discrimination dans l'enseignement de l'histoire aussi bien que des expressions de résistance à ses constructions discursives, des manifestations qui permettent de comprendre le rôle stratégique que l'histoire a joué comme discipline scolaire, dans le processus de scolarisation, pour la construction de l'hégémonie/subalternisation, à l'intérieur d'un contexte inter-ethnique mapuche/non mapuche.

ENSEIGNEMENT DE L'HISTOIRE • L'IDENTITÉ • ELÈVE • CULTURE MAPUCHE

\section{A IDENTIDADE NEGADA: HISTÓRIA E SUBALTERNIZAÇÃO CULTURAL A PARTIR DE TESTEMUNHOS ESCOLARES MAPUCHE \\ RESUMO}

Este artigo apresenta resultados de uma pesquisa cujo objetivo foi o de indagar experiências escolares de pessoas mapuche, especificamente no tocante às suas significações com relação à história ensinada em sua formação escolar. A pesquisa socioeducativa foi desenvolvida a partir de uma perspectiva histórica, utilizando a revisão documental como técnica de produção de informação e a análise de conteúdo como procedimento para reduzir e categorizar a informação relevante. Os resultados mostram experiências de agravo e discriminação na história ensinada, bem como expressões de resistência às suas construções discursivas, todas elas manifestações que permitem compreender o papel estratégico da história como disciplina escolar, no processo de escolarização, para a construção de hegemonia/subalternização no contexto interétnico mapuche/não mapuche. 

educativo tan estratégico como polémico. Es por ello que a fuerza de cuestionamientos y debates curriculares se han levantado renovadas propuestas acerca del rol pedagógico e importancia del saber histórico como conocimiento escolar, superadoras de los propósitos nacionalistas primigenios que caracterizaron su enseñanza.

Las orientaciones curriculares actuales de la historia escolar, tanto en Europa como en América Latina, presentan propósitos formativos vinculados a la promoción de una ciudadanía democrática, al respeto de los derechos humanos, al desarrollo de habilidades cognitivas de indagación e interpretación de información, así como del pensamiento crítico y reflexivo (COX et al., 2009; TOLEDO; GAZMURI; MAGENDZO, 2010). También, en el contexto del reconocimiento de la multiculturalidad de las sociedades, las orientaciones curriculares promueven la valoración de la diversidad cultural como una forma de eliminar males sociales como el etnocentrismo y el racismo (PRATS; BARCA; FACAL, 2013), y la afirmación identitaria de las subjetividades escolarizadas.

Estos renovados propósitos formativos se corresponden con el reconocimiento de los derechos educativos y culturales de los pueblos originarios en América Latina, cuestión que pareciera augurar una nueva relación educativa entre el sistema escolar y las comunidades originarias respecto de la historia enseñada. Sin embargo, no se puede obviar aquel conocimiento educativo que ha planteado la distancia que existe entre 
el currículum prescrito y el desplegado en el aula, y aquel que propone que el currículum es una construcción social y simbólica que surge de la interacción entre los diversos sujetos y actores educativos en sus contextos. Menos el que informa acerca de las tensiones y complejidades que conllevan los procesos formativos en espacios sociales y educativos interétnicos, dados los posicionamientos y epistemes diferenciadas que operan en estos contextos.

Más allá de las complejidades educativas enunciadas, en Chile la construcción de lo educativo e histórico en contexto mapuche se ha configurado en el marco de la conflictiva relación del Estado chileno con el pueblo originario, o para ser más preciso, en un contexto de relaciones interétnicas violentas y asimétricas. Ello nos permite levantar la hipótesis comprensiva que orienta el presente estudio: la historia como disciplina escolar en contexto interétnico mapuche/no mapuche promovió/promueve un discurso pedagógico que excede a una concepción tradicional de su enseñanza, configurando, más bien, procesos de exclusión y subalternización cultural que buscó/busca negar la identidad cultural originaria.

El artículo presenta los resultados de una investigación que tuvo como objetivo recuperar, en perspectiva histórica, testimonios de experiencias educativas en clases de historia de personas mapuches que asistieron a la escuela chilena en el contexto interétnico. Se recogen relatos escolares de educadores e intelectuales mapuche expuestos en fuentes escritas como Memorias de Titulación, artículos científicos, periódicos, entre otros, y en términos temporales comprende testimonios de mediados del siglo pasado y otros de data reciente. Es por tanto, una investigación documental, en perspectiva histórica y geográficamente situada en la Araucanía, en el territorio tradicional mapuche (región centro-sur de Chile).

\section{MARCO TEÓRICO}

\section{HISTORIA Y FORMACIÓN DE IDENTIDAD}

En un estudio referido a la función social de la historia, Florescano (2013) plantea que dotar de identidad a un pueblo, proveerlo de un relato común acerca del pasado y una memoria histórica formadora de sentidos compartidos, es la más antigua y constante función social de la historia. Para ello, la memoria histórica se encarga de delimitar el campo de la vida social propia, separándola de la vida de los "otros". Aquello explicaría -según Florescano- el interés y atractivo por el relato histórico, así como la vasta y continua renovación de su audiencia.

Esta función también es expuesta por Villoro cuando estipula que "ninguna actividad intelectual ha logrado mejor que la historia dar conciencia de la propia identidad a una comunidad” (VILLORO, 1980, p. 44). 
En el mismo sentido se puede leer la respuesta a la pregunta ¿para qué se estudia la historia?, planteada por el historiador británico Collingwood; su estudio -propone- es una indagación acerca del significado de la vida individual y colectiva de los seres humanos a través del tiempo.

Pareciera ser que la historia constituye una eficaz herramienta para hacernos partícipe de una comunidad de referencia y discriminar entre "nosotros" y los "otros". No obstante, la historia entendida como disciplina científica excede con creces, en la actualidad, a las preocupaciones identitarias primigenias, cuestión que se refleja en la ampliación del orden temático de estudio, en la emergencia de nuevos sujetos históricos e identidades socioculturales, y en la propia complejidad con que se estudia y discute el concepto de identidad. El rezago se presenta más bien en la historia enseñada o en su versión escolar.

Un rasgo distintivo de las sociedades actuales es su carácter multiétnico, dada la coexistencia en un mismo espacio socio-territorial de diferentes grupos étnicos que dan origen a relaciones interétnicas e interculturales. Las primeras se refieren a las relaciones entre grupos que se diferencian y reconocen en su identidad étnica, en sus sistemas simbólicos como la lengua y la religión, entre otras; las segundas involucran relaciones e interacciones entre grupos humanos diferentes, donde se entrecruzan sistemas simbólicos generando transformaciones culturales entre los grupos que se relacionan (LOSLIER, 1997). Estas nuevas configuraciones sociales, así como el reconocimiento de la diversidad cultural y la presencia de la alteridad en la escuela, interpelan la matriz monocultural/eurocéntrica de la historia escolar, cuestión que para Rodríguez Ledesma (2009) conlleva una necesaria renuncia a los viejos y consolidados conceptos, nociones y periodizaciones, universalismos y nacionalismos para dar espacio a la construcción de una historia escolar abierta a diversas voces y saberes educativos, de manera que otras identidades culturales presentes en la escuela puedan visibilizarse y/o emerger de los procesos formativos.

\section{IDENTIDAD NACIONAL Y SUBALTERNIZACIÓN CULTURAL}

La investigación acerca de la historia enseñada ha evidenciado el rol privilegiado que cabe a esta disciplina escolar en la formación de identidades patrias, en el contexto de la conformación de los sistemas educativos nacionales. Quienes, desde el currículo prescrito, asumen una concepción tradicional de su enseñanza, proponiendo como objetivos educativos de la historia escolar la formación de ciudadanos impregnados de amor a la patria, cultos y respetuosos de las instituciones, 
autoridades y las leyes (GOJMAN, 2001; CARRETERO, 2007; TOLEDO; GAZMURI; MAGENDZO, 2010).

Este discurso pedagógico de la historia se construyó, como política educativa estatal, en base a un repertorio de imágenes heroicas e instalación de jerarquías que recreaban la trayectoria patria y una representación del pasado social como constitutiva de una "comunidad cultural” y tuvo como propósito explícito interiorizar en las cohortes estudiantiles el sentido de pertenencia a una nación, una cultura común y a un orden estatal (TURRA-DÍAZ, 2015).

Empero, la historia en su registro escolar aún promueve un relato común del pasado, como plantea Carretero (2007), predominando en ella objetivos románticos que guardan una íntima vinculación emotiva con los símbolos y los relatos de identidad nacional, siendo los objetivos de orden cognitivo y de pensamiento crítico relegados a un segundo plano. Se trata de una narrativa que tiene como finalidad educativa prioritaria la proyección "de una imagen positiva, triunfal, progresista" de la historia nacional, para de esta manera formar ideológicamente a las nuevas generaciones a través de la escuela (CARRETERO, 2007, p. 38).

Por su parte, la historia de la educación ha visibilizado la estrecha relación entre el surgimiento del Estado Liberal, la escolarización y la enseñanza de la historia, así como su rol estratégico en la invención de la comunidad nacional. Ello significa que las múltiples identidades que se producen en el entramado de lo social y cultural son sometidas a una con pretensión de totalidad y hegemonía, como es el caso de las identidades nacionales que se promueve en el seno de las sociedades modernas. Una comunidad nacional "imaginaria e imaginada", en términos de Anderson (1993), puesto que buena parte de los sujetos que la componen no se conocen entre sí, y solo reconocen a los otros nacionales en la medida que portan una imagen de pertenencia común, construida tempranamente en el sistema escolar. En esta perspectiva, nación e identidad nacional se configuran como una realidad construida, «objetivada», y en contenido de la socialización del individuo.

Evidentemente, esta lógica de construcción de comunidad operó/opera en base a una dialéctica de inclusión/exclusión que, para el caso de América Latina, determinó que los pueblos originarios adquieran la calidad de “otros”. En este propósito, la historia escolar despliega un especial protagonismo en la producción y difusión de un conocimiento histórico de cuestionable cientificidad, que promueve una representación devaluada de las formas culturales de los pueblos originarios por medio de distorsiones, estereotipos y caricaturización de sus modos de vida (AMAN, 2010), expresiones que dan cuenta, abiertamente, de un racismo discursivo (VAN DIJK, 2007).

Como contrapartida y consecuencia de la hegemonía discursiva, se instituyó un proceso de subalternización cultural, en términos de 
Guha (1988, 1996), en donde el subalterno se ubica en un espacio marginal del desarrollo histórico y desde una posición subordinada. Para el caso, la subalternidad debe entenderse como atributo de la subordinación, siendo la identidad del subalterno la negación.

Este proceso es el que han vivido los pueblos originarios, como consecuencia del menoscabo e inferiorización de sus formas de vida proyectados por el discurso histórico, que niega la construcción de identidad social y cultural a través de los procesos formativos escolares.

El reconocimiento y oficialización de los derechos educativos de los pueblos originarios buscan superar la dialéctica de negación del “otro" indígena en los procesos formativos escolarizados y con ello, nuevamente, la enseñanza de la historia adquiere un rol privilegiado en la conformación de subjetividades abiertas e interculturales. No obstante, todo proceso que busque construir nuevos sentidos en la enseñanza de la historia en contextos interétnicos implica historizar su propio despliegue, relevar sus discursos y sus propósitos formativos. También implica reconocer los posicionamientos étnicos, las experiencias y trayectorias educativas, que permitan develar la violencia cultural y las asimetrías que se proyectaron en el espacio interétnico y que constituyen el corpus simbólico que portan los sujetos que habitan estos espacios.

\section{METODOLOGÍA}

El estudio se desarrolló desde una metodología de investigación histórica, por cuanto su información fundamental se generó desde la revisión documental. Para el caso, nos servimos de diversos trabajos que contienen testimonios de personas mapuches, ya sea como relatos directos en sus propios escritos (Memoria de Título, escritos de divulgación) o de entrevistas recogidas por otros autores en artículos científicos.

También se utilizó el estudio documental de Foerster y Montecino -Organizaciones, líderes y contiendas mapuches (1900-1970) (1988)-, que recoge testimonios contenidos en la prensa local, el "Diario Austral de Temuco". De los documentos de época se seleccionaron testimonios y declaraciones que aportan al objetivo del estudio, y que permitieron configurar las categorías de demandas levantadas.

La concentración de significados comunes expresa las categorías o unidades de análisis y de ellas se presentan los relatos más representativos como sus resultados.

\section{RESULTADOS}

DE AGRAVIOS Y DISCRIMINACIÓN EN LA ENSEÑANZA DE LA HISTORIA

Uno de los primeros relatos de experiencia en la enseñanza de la historia en escuelas de la Araucanía es aquel que expone la educadora Zenobia Quintremil en su Memoria de titulación como profesora normalista, el año 1948: 
Ninguna profesora en ninguna clase de historia, siquiera en cumplimiento de un deber estricto, hizo notar que la raza chilena es el producto lógico del choque tremendo, que se prolongó por tres siglos, de dos razas formidables: la española y la araucana, la conquistadora y la inconquistada... No abrigo el rencor ni mucho menos el odio. Por el contrario, desde aquí, desde estas líneas que acaso permanecerán ignoradas, perdono todas las ofensas y agravios que me han inferido y que, indudablemente, han debido sufrir quienes llevan la noble y limpia sangre de la raza araucana. (FOERSTER; MONTECINO, 1988, p. 269)

El testimonio da cuenta de los padecimientos que desde la historia escolar se profería al estudiantado mapuche a causa de una enseñanza que buscaba, a fuerza de agravios y ofensas, inculcar la inferioridad de la cultura del pueblo originario y con ello legitimar el papel subordinado que le correspondía en el contexto de la sociedad chilena a la que debía asimilarse, a través de la acción educativa de la escuela. También, la adopción de una identidad social y racial promovida desde la cultura hegemónica, la de araucano y de raza araucana, que un sector de la sociedad originaria asumió acríticamente, especialmente los de mayor escolarización de aquella época.

Los contenidos de enseñanza estaban cruzados por representaciones y estereotipos que recogían los conceptos e ideas levantadas por la historiografía decimonónica y aquella que se estaba forjando en el siglo XX, instituyéndose abiertamente en expresiones discriminatorias para el estudiantado, como testimonia el siguiente relato escolar de mediados de aquel siglo:

\footnotetext{
Pero en cuanto a la educación que entregaban en ese tiempo era de que, referente a los mapuches, nos enseñaban a nosotros de que los indios eran semisalvajes y por lo tanto se vestían con pieles y comían la carne, que sé yo, semisancochada, y andaban descalzos y todo ese tipo de cosas, entonces como que éramos un pueblo muy retrasado según lo que nos educaban a nosotros en ese colegio. (POBLETE, 2003, p. 60)
}

El relato evidencia una narrativa histórica cargada de representaciones sociales estereotipadas que, como efecto mínimo, provocaba en el estudiantado mapuche una experiencia de discriminación percibida, pero que a largo plazo se constituye en un factor clave en la desvalorización cultural y conformación de identidades estigmatizadas. Asimismo, se pone en evidencia la identidad genérica asignada a la población originaria "Latinoamericana”, la de indio, proyectada hasta décadas recientes. 
La historia enseñada en las escuelas de la Araucanía comprendía conceptos, denominaciones e imaginarios respecto del pueblo mapuche que tensionaban la identidad cultural de los jóvenes aprendices, pues se trataba de un lenguaje ajeno y ofensivo para con su cultura y sociedad de referencia, como lo expresa una reconocida académica mapuche al recordar su paso por la escuela:

Cuando a los 10 años sali de mi comunidad a estudiar al pueblo, senti el peso de otro lenguaje que caía sobre mi y que me marcó profundamente. En las clases de historia [...] se repetían las palabras india, indio o araucano y se decía que ya no existíamos... Yo escuchaba callada en la sala, sentía culpas de ser y de estar alli; tenía conciencia de ser mapuche porque de mis padres aprendi ese orgullo, pero en la escuela vivía con profunda tristeza y soledad esta condición. (Entrevista a Elisa Loncon, 2011)

En general, los relatos dejan de manifiesto experiencias de agravio y sufrimiento en el aprendizaje de historia, por cuanto se promovía una enseñanza que degradaba las formas culturales tradicionales mapuche y abiertamente declaraba extinto e inexistente a este pueblo originario. Esto ocurría en un tiempo, segunda mitad del siglo XX, en que las políticas públicas educacionales intensificaron la cobertura educacional en el contexto interétnico y la obligatoriedad de la enseñanza exigía a las familias mapuches a enviar a sus hijos e hijas a la escuela chilena que se expandía en la Araucanía. Desde el punto de vista formativo, la historia enseñada tuvo su interés principal en generar aprendizajes actitudinales, en la adquisición de valores y sentimientos vinculados a la chilenidad a la cual debían incorporarse.

Si bien las reformas curriculares de fines de los noventa introdujeron orientaciones de enseñanza de la historia tendientes a reconocer la diversidad cultural, investigaciones recientes en historia escolar recogen testimonios que muestran una persistencia de situaciones de discriminación étnica en las prácticas de enseñanza, como expresa un relato de un estudiante perteneciente a la identidad territorial mapuche-pewenche:

Yo soy hablante de chedungun... esas cosas que pasan en historia, como por ejemplo, ya mataron a un indígena, a mí me empieza a dar rabia una cosa así... creo que hay muchas contradicciones entre chilenos y nosotros, la clase de historia para mí sería mejor, yo la dejaría de lado, porque empiezan como a discriminarse... me da mucha rabia que me digan indígena, prefiero que me digan mapuche o pewuenche, pero indígena, lo escuchamos mucho en la clase y uno tiene que guardarse la opinión. (PARRA; PICHIÑUAL, 2012, p. 27) 
La narrativa histórica degradadora de la imagen del pueblo mapuche no resultó indiferente para los profesores y jóvenes intelectuales de la etnia que egresaban del sistema educativo chileno por lo que, tempranamente, levantaron sus voces para denunciar esta anomalía educativa. Así, la organización Juventud Moderna Araucanía, por acuerdo de una asamblea efectuada el año 1943, en Temuco, expresaba como una de sus peticiones:

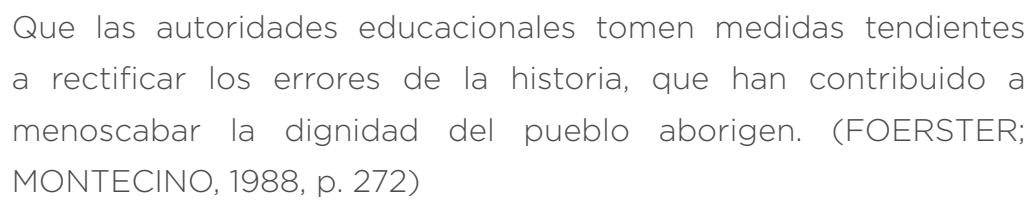

Los errores de la historia se refieren a la proliferación de discursos e imaginarios levantados desde diversos estudiosos sociales, que promovían una representación de la Araucanía y sus habitantes ancestrales en base a una dialéctica exclusora, en donde lo mapuche se asociaba con la barbarie. Importante rol cabe a la historiografía decimonónica en la construcción de esta narrativa degradadora del pueblo mapuche, en particular a dos de sus máximos exponentes, Vicuña Mackenna y Barros Arana, quienes desde una concepción positivista de la historia categorizaron a la población mapuche como seres de segunda categoría, presentándolos como peligrosos salvajes o en el mejor de los casos como indómitos bárbaros (BELLO, 2004; CHIHUAILAF, 2012). También la obra del ensayista y educador Tomás Guevara se esfuerza en demostrar, con pretensión de cientificidad, la "mentalidad pre-lógica" que caracterizaría a este pueblo, situación que hacía del todo justificable la ocupación de su territorio y la incorporación de sus habitantes originarios a la civilización representada por la sociedad chilena (SALAS, 2009).

Por cierto, la narrativa historiográfica que configuró una imagen degradada del pueblo mapuche se instituyó como hegemónica y, prontamente, transitó hacia el campo educativo, oficializándose como contenido de enseñanza de la historia escolar, encubriendo con ello otro fenómeno histórico: la ocupación violenta del territorio mapuche por parte del estado chileno. El crítico testimonio de un reconocido ensayista mapuche ilustra esta suerte de tradición discursiva en la enseñanza de la historia: 
como desinformado. Tras siglos de querra con España, los araucanos finalmente aceptaron el llamado de la civilización y el progreso. Y en la cumbre del cerro Nielol, en una jornada histórica, sellaron con las autoridades chilenas la paz definitiva... Huelga decir que la versión de mi abuelo Alberto era completamente distinta. Él, que había pasado media vida en tribunales reclamando inútilmente tierras usurpadas, sabía que de mosto y música el arribo chileno había tenido bastante poco. Y si mucho de pólvora. Y de saqueos. Y corridas de cerco. (CAYUQUEO, 2010)

En el contexto de las actuales demandas educativas del pueblo mapuche, promovidas desde un enfoque de derechos educativos y culturales de los pueblos originarios, la persistencia de discursos nacionalistas y racistas en la enseñanza de la historia, que actualiza antiguas ideologías y prácticas socioeducativas, parece no ser tolerada por las nuevas generaciones de mapuches y, más aún, se avanza en evidenciar la desconexión existente entre la historia enseñada y la historia vivida por la comunidad, como se puede leer en el testimonio citado.

$\mathrm{Al}$ mismo tiempo, emergen voces de educadores e intelectuales mapuches que reclaman para las nuevas generaciones de estudiantes indígenas el derecho de acceder a su patrimonio histórico-cultural en el sistema escolar, a formarse con sus propias narrativas históricas. Aquello implica colocar en cuestión y manifestar su resistencia a una enseñanza de la historia de orden monocultural, que excluye la episteme y la memoria histórica indígena de sus contenidos pedagógicos, como se puede leer en los párrafos siguientes:

Un joven me preguntaba por qué no se cuenta que del Biobio al sur era un país diferente que fue invadido en la historia de Chile. ¿Por qué no se cuenta eso en los libros de historia. Hoy día están apareciendo esas cosas, pero no intencionadas desde el estado, sino desde organismos internacionales que han dado a conocer los tratados. No es el país a través de los mismos colegios el que pueda impartir esta información porque, al fin y al cabo, esta información es dar a conocer un saber, un conocimiento. (NAÍN CURAMIL, 2014)

En Chile hay dos historias que caminan de forma paralela: la del pueblo chileno y la del pueblo mapuche, aunque ambas son tan importantes, nunca se han juntado [...] la historia mapuche y en general la de todos los pueblos indígenas no está en la educación formal, no está dentro de los libros de historia [...]. Pasarán muchos 


\section{DISCUSIÓN}

Una primera idea a discutir es la función social que asume el relato histórico escolarizado en el contexto interétnico. Es evidente que su propósito fundamental era la formación de subjetividades chilenonacionales, por lo que lejos estaba una función social de la historia que aportara a la construcción de una identidad étnica que recogiera las formas culturales de la sociedad mapuche. La historia en la escuela interétnica tiene como responsabilidad chilenizar a las cohortes estudiantiles mapuche/no mapuche a través de un relato que fomentaba la positiva valoración del pasado, presente y futuro de la trayectoria patria y que estaba por encima de cualquier diferencia étnica, cultural o lingüística. En este propósito, los escolares mapuche eran sometidos a una especie de coerción ideológica en la que, a fuerza de repetición y memorización, se esperaba que internalizaran los relatos heroicos, valores y normas que daban forma y contenido a la nación chilena. En esta perspectiva, la historia escolar se puede representar como una manifestación discursiva del colonialismo interno, tomando como referencia el potencial analítico del concepto acuñado por González Casanova (TORRES GUILLEN, 2014), en tanto configura y distribuye un conocimiento criollo-estatal que, por un lado, niega y barbariza las formas de ser y pensar del pueblo originario mapuche y, por otro, impone la tradición cultural europeo-occidental en cuya matriz se inscribiría -según el relato configurado- la trayectoria histórica de la chilenidad.

Un segundo aspecto a discutir se refiere a los agravios y la discriminación percibida por las subjetividades mapuche en su experiencia escolar. Claramente, ello es el reflejo de la violencia simbólica expresada en el relato histórico, que en su afán de negar y omitir todo aquello que no es útil a la formación de nacionales configura al mapuche como un "otro" cultural, aunque perteneciente a una sociedad extinta y/o, en el mejor de los casos, diluido en un proceso de mestizaje que invisibiliza su existencia contemporánea. Proclamar su desaparición como pueblo y cultura implicaba eliminar una amenaza para la homogeneidad cultural, pero a la vez genera una toma de conciencia del colonialismo en la persona mapuche, así como también una conciencia étnica-mapuche (DURÁN; CATRIQUIR; BERHO, 2011), pues visibiliza el antagonismo cultural y los estereotipos configurados respecto de la cultura originaria, cuestión que los lleva a levantar mecanismos de resistencia discursiva que se expresan en una resistencia a asumir la identidad chilena y de paso reivindicar la identidad cultural originaria.

El Estado Chileno, a través del proceso de escolarización en la Araucanía, desplegó procesos de dominación cultural en los que la enseñanza de la historia viene a cumplir un papel fundamental en la construcción de identidad nacional en un contexto de diferencia étnica 
y cultural. Por lo mismo, actuó como un potente dispositivo simbólico generador de procesos de exclusión y subalternización cultural.

Desde la historización de la enseñanza de la historia es posible aportar a la transformación de una historia escolar, concebida como dispositivo curricular de construcción de hegemonía étnico-cultural -los testimonios recientes nos llevan a sospechar que su enseñanza sigue anclada en lo fundamental en estos propósitos- hacia una historia escolar entendida como una operación discursiva y analítica que comprenda las diferencias culturales, sociales y étnicas desde una perspectiva intercultural, democrática y dialógica con las comunidades y los territorios. Lo anterior implica un escenario abierto para los sujetos mapuches y no mapuches a construir sus propios procesos de identificación con la nación y la comunidad. Dicho lo cual la enseñanza de la historia, en contexto interétnico, debiese entregar herramientas para que los sujetos tomen decisiones sobre un modo de ser, pensar y estar en un territorio determinado; esto implica propender a la creciente autonomía curricular de las comunidades en pro de establecer un dialogo con la escuela chilena, sin prejuicios ni pretensiones de superioridad y hegemonía. Es decir, una historia enseñada y construida desde lo mapuche, que fortalezca su propia historia, alimente la tradición cultural y se transforme en una herramienta de autonomía y control cultural, a la manera de Bonfil Batalla (1991). Por cierto, para aquello es relevante la presencia de un profesorado reflexivo capaz de cuestionar la naturalización de los saberes escolares, quien puede aportar a una enseñanza dialógica que resignifique el sentido de los contenidos de su enseñanza y permita un abordaje pedagógico de las tensiones de las sociedades en contacto y de la propia sociedad chilena.

\section{CONCLUSIONES}

La trayectoria de la enseñanza de la historia en la educación chilena ha estado orientada fundamentalmente a la formación de un tipo ideal nacional que ha consolidado la idea de una nación hegemónica e invariablemente unida a una historia y origen común. En la Araucanía histórica, la escolarización y la enseñanza de la historia operaron como una verdadera cruzada ideológica colonialista que dio origen a profundos antagonismos culturales -proyectados hasta la actualidad- y que en su contenido promovió un relato teñido de discriminación y racismo, orientados al menoscabo e inferiorización del pueblo mapuche.

Los testimonios escolares dan cuenta de los contenidos y efectos simbólicos que conlleva una historia escolar monocultural y exclusora, en tanto dispositivo de poder constructora de hegemonía/subalternidad, que en el caso de la sociedad mapuche y la chilena ha generado profundas atrofias culturales cuyas huellas y manifestaciones de resistencia se pueden rastrear hasta el presente. Por ello, entendemos como una 
necesidad historizar la historia escolar, pero también transformar su impronta monocultural, pues cualquier posibilidad de avanzar en la construcción de sentidos y prácticas interculturales comienza por visibilizar la reconocida conflictividad étnica y discursiva instituida en el espacio escolar y que genera asimetrías educativas. Implica reconocer los diversos posicionamientos étnicos, las trayectorias culturales, y desde ahí configurar las diversas narrativas históricas que otorguen sentido y fundamentos a los sujetos que habitan el espacio interétnico.

\section{BIBLIOGRAFÍA FUENTES DOCUMENTALES}

CAYUQUEO, Pedro. ¿Menos clases de historia?, Bienvenido sea. The Clinic, 25 nov. 2010. Disponible en: <www.theclinic.cl>. Acesso em: 25 nov. 2010.

FOERSTER, Rolf; MONTECINO, Sonia. Organizaciones, líderes y contiendas mapuches (1900-1970). Santiago de Chile: CEM, 1988.

HUIANAO, Graciela. Entrevista. Fûtawillimapu Espacio Mapuche williche en Internet, Grande Território Sul Mapuche, jul. 2012. Disponible en: <http://www.futawillimapu.org/Llitu/ Entrevista-Graciela-Huinao-poetisa-No-me-siento-chilena-porque-yo-naci-mapuche.html> . Acesso em: ago. 2015.

LONCÓN, Elisa. Entrevista. El Ciudadano, Santiago de Chile, n. 108, 15 ago. 2011. Disponible en: <www.elciudadano.cl>. Acesso em: 15 ago. 2011.

NAÍN CURAMIL, José. La conciencia nace del sufrimiento y del saber: experiencia como educador tradicional mapuche en la comunidad de Temucuicui. Revista Vínculos, Santiago de Chile, n. 1, 2014. Disponible en: <http://revistavinculos.cl/ediciondigital/project/la-conciencianace-del-sufrimiento-y-del-saber-experiencia-como-educador-tradicional-mapuche-en-lacomunidad-de-temucuicui/>. Acesso em: feb. 2015.

PARRA, V.; PICHIÑUAL, A. Escolares Mapuche-Pehuenche en la clase de historia. 2012. Tesis (Doutorado) - Universidad de Concepción, Concepción, 2012.

\section{FUENTES BIBLIOGRÁFICAS}

AMAN, Robert. El indígena "latinoamericano" en la enseñanza: representación de la comunidad indígena en manuales escolares europeos y latinoamericanos. Estudios Pedagógicos, v. 36, n. 2, p. 41-50, 2010.

ANDERSON, Benedict. Comunidades imaginadas: reflexiones sobre el origen y la difusión del nacionalismo. Buenos Aires: Fondo de Cultura Económica, 1993.

BELLO, Alvaro. Los mapuches de Chile entre la historia y el mito. Revista de Educación Aula Abierta, año 10, p. 131-132, 2004.

BONFIL BATALLA, Guillermo. La teoría del control cultural en el estudio de procesos étnicos. Estudios sobre las Culturas Contemporáneas, v. 4, n. 12, p. 165-204, 1991.

CARRETERO, Mario. Documentos de identidad. La construcción de la memoria histórica en el mundo global. Buenos Aires: Paidós, 2007.

CHIHUAILAF, Arauco. La representación de los mapuches en la historiografía chilena: 1882-1973. In: CANCINO, Hugo et al. (Ed.) Miradas desde la Historia social y la Historia intelectual. América Latina en sus culturas: de los procesos independentistas a la globalización. Córdoba: Centro de Estudios Históricos, 2012. p. 467-479.

COX, Cristián; LIRA, Robinson; GAZMURI, Renato. Currículos escolares y sus orientaciones sobre historia, sociedad y política: significados para la cohesión social en Latinoamérica. In: COX, C; SCHWARZTMAN, S. (Ed.). Políticas educativas y cohesión social en América Latina. Santiago de Chile: Uqbar, 2009. p. 231-288. 
DURÁN, Teresa; CATRIQUIR, Desiderio; BERHO, Marcelo. Diversidad cultural e interculturalidad en una Universidad del centro Sur de Chile. Validando una categoría analítica. Cuadernos Interculturales, v. 9, n. 17, p. 135-159, 2011.

FLORESCANO, Enrique. La función social de la Historia. México DF: Fondo de Cultura Económica, 2013.

GOJMAN, Silvia. La historia: una reflexión sobre el pasado: un compromiso con el futuro. AISEMBERG, B.; ALDEROQUI, S. (Comp). Didáctica de las ciencias sociales. Aportes y reflexiones. Buenos Aires: Paidos, 2001. p. 42-62.

GUHA, Ranajit. Dominance without Hegemony: history and power in Colonial India. Delhi: Harvard University Press, 1988.

GUHA, Ranajit. The small voice of history. In: GUHA, Ranajit. Subaltern Studies. Delhi/Bombay/ Calcutta: Oxford University Press, 1996.

LOSLIER, Sylvie. Des relations interculturelles: du roman à la réalité. Quebec: Liber, 1997.

POBLETE, María Pía. Discriminación étnica en relatos de la experiencia escolar mapuche en Panguipulli, Chile. Estudios Pedagógicos, n. 29, p. 55-64, 2003.

PRATS, Joaquín; BARCA, Isabel; FACAL, Ramón. Historia e identidades culturales. Braga: Universidade do Minho, 2013. Disponible en: http://webs.ie.uminho.pt/conscienciahistorica/ Historia_Identidades_culturales.pdf>. Acesso em: ene. 2014.

RODRÍGUEZ LEDESMA, Ximena. Historia y diversidad. El lugar de la otredad en la reconversión del paradigma imperante. Cuadernos México, v. 1, p. 29-38. 2009.

SALAS, Ricardo. Civilización e historia en A. Comte y T. Guevara. In: MUÑOZ, M.; VERMEREN, P. (Ed.). Repensando el siglo XIX desde América Latina y Francia: homenaje al filósofo Arturo Andrés Roig. Buenos Aires: Ediciones Colihue, 2009. p. 205-216.

TOLEDO, María Isabel; GAZMURI, Renato; MAGENDZO, Abraham. Propuestas de categorización de corrientes vigentes en la enseñanza escolar de la historia. Iber: Didáctica de las ciencias sociales, geografía e historia, n. 63, p. 77-92, 2010.

TORRES GUILLEN, Jaime. El carácter analítico y político del concepto de colonialismo interno de Pablo González Casanova. Desacatos, n. 45, p. 85-98. 2014. Disponible en: <http://www.scielo. org.mx/pdf/desacatos/n45/n45a8.pdf>. Acesso em: ene. 2015.

TURRA-DÍAZ, Omar. Profesorado y saberes histórico-educativos mapuche en la enseñanza de historia. Revista Electrónica EDUCARE, v. 19, n. 3, p. 1-20. 2015. Disponible en: http://www. revistas.una.ac.cr/index.php/EDUCARE/article/view/6879/7085>. Acesso em: nov. 2015.

VAN DIJK, Teum. Racismo y discurso en América Latina. Barcelona: Gedisa. 2007.

VILLORO, Luis. El sentido de la historia. In: PEREYRA Carlos et al. Historia ¿para qué? México DF: Siglo XXI, 1980. p. 35-52.

\section{OMAR TURRA}

Universidad del Bío Bío, Chillán, Chile

oturra@ubiobio.cl

DESIDERIO CATRIQUIR

Universidad Católica de Temuco, Temuco, Chile

dcatri@uct.cl

MARIO VALDÉS

Universidad de Concepción, Concepción, Chile

mariovaldes@udec.cl 